\title{
PAOLO MANTEGAZZA: RETTORE DELL'UNIVERSITÀ DEGLI STUDI DI MILANO
}

\author{
GIANPIERO SIRONI (*)
}

SunTo. - Paolo Mantegazza è stato Rettore dell'Università degli Studi di Milano per quattro mandati consecutivi, dall'anno 1984 al 2001, dopo essere stato per 10 anni Preside della Facoltà di Medicina e Chirurgia (dal 1974 al 1984). Il periodo del suo Rettorato è stato un periodo di grandi cambiamenti, nella società, ma anche nell'università, in seguito alla legge di riforma universitaria, il DPR 382, varata nel 1980. Nel 1989 la legge n. 168 concesse alle università l'“autonomia” statutaria e regolamentare prevista dall'art. 33 della Costituzione. In quegli anni si è avuto inoltre un grande aumento della popolazione studentesca, in particolare dell'Università di Milano. Mentre all'inizio del Rettorato di Paolo Mantegazza, nell'anno accademico 1983-84, vi erano 63.450 studenti iscritti, il numero di studenti è nel corso degli anni pervenuto ad un numero vicino a 100.000 , comportando un intenso lavoro per rendere adeguato l'espletamento della didattica e l'attività complessiva dell'Ateneo per un così grande numero di studenti. Paolo Mantegazza ha guidato l'Università tutta per cercare di assolvere questo compito crescente, agendo su più fronti. Tra questi anche in relazione al quadro normativo complessivo dell'Ateneo. Infatti egli ha promosso il varo di un nuovo Statuto dell'Ateneo (1996) e l'approvazione dei principali regolamenti da esso previsti, dando attuazione all'autonomia dell'Ateneo. La crescita dell'Ateneo pose problemi di diversa natura: di aumento delle dimensioni del corpo docente, per far fronte al grande numero di studenti, ma anche di nuove necessità edilizie. Le sue realizzazioni su quest'ultimo fronte sono state molto numerose, in ambiti molto diversi, nell'interesse della maggior parte delle Facoltà dell'Ateneo. E tuttavia le dimensioni raggiunte dall'Università di Milano richiedevano un piano di più ampia portata per attuare il decongestionamento dell'Ateneo. Si puntò sul raddoppio di alcune Facoltà e sull'istituzione di nuovi corsi di laurea e di nuove Facoltà. Fu tuttavia ben presto chiaro che la soluzione più razionale consisteva nella istituzione di un secondo Ateneo statale a Milano, ipotizzato già in occasione della redazione del Piano triennale 1991 -1993. Nacque così, nel 1998, l'Università di Milano Bicocca. Nello stesso anno venne istituita anche l'Università

(*) Istituto Lombardo Accademia di Scienze e Lettere, Università degli Studi di Milano, Italia. E-mail: gianpiero.sironi@unimi.it 
dell'Insubria, con sedi a Como e Varese. A tale istituzione Paolo Mantegazza e l'Università di Milano contribuirono, insieme con l'Università di Pavia. Nel 1996 venne istituita anche l'Università Vita e Salute San Raffaele, alla cui crescita l'Università di Milano aveva notevolmente contribuito durante il rettorato di Paolo Mantegazza, distaccando in quella sede numerosi docenti della Facoltà di Medicina e Chirurgia. Senza dimenticare il ruolo che Mantegazza aveva avuto come Preside di Medicina nel favorire un insediamento universitario a Brescia e la successiva costituzione, nel 1982, di una università autonoma. L'impegno di Mantegazza per lo sviluppo della ricerca scientifica in ateneo è stato davvero a tutto campo. Al termine del suo Rettorato l'Università di Milano risultava essere la prima università italiana in diverse classifiche internazionali relative alla produzione scientifica. Fu principalmente sulla base di tali risultati che, al termine del suo Rettorato, nell'anno 2002, l'Università di Milano, unica università italiana, veniva invitata ad essere una delle dodici università europee chiamate a costituire la "League of European Research Universities" (più nota con il suo acronimo: LERU), che collega tra loro Università europee di grande prestigio. Durante il suo Rettorato egli ricoprì (per due successivi mandati) anche il ruolo di VicePresidente della Conferenza dei Rettori delle Università Italiane. Paolo Mantegazza fu un Rettore di notevoli qualità umane, con una grande capacità operativa, unita ad un carattere mite. Ebbe una grande attenzione agli studenti ed alle loro esigenze, considerando che l'insegnamento e la formazione degli studenti fossero lo scopo primario dell'Università. Poco dopo la conclusione del suo Rettorato l'allora Ministro dell'Istruzione, dell'Università e della Ricerca, Letizia Moratti, accolse la modifica dello Statuto dell'Università che nel frattempo era stata proposta, con l'aggiunta di un nuovo articolo (Art. 56), che così recita: "In considerazione dei meriti acquisiti e dell'impegno determinante profuso per l'Ateneo nel corso del suo Rettorato, al prof. Paolo Mantegazza è conferito il titolo onorifico di Rettore emerito".

ABSTRACT. - Paolo Mantegazza has been Rector of the University of Milan for four consecutive mandates, from 1984 to 2001, after having been for 10 years Dean of the Medicine and Surgery Faculty (from 1974 to 1984). His rectorship period has been a time of great change in the society but also in university, as a result of the University reform law, Decree 382, passed in 1980. In 1989 the law no. 168 granted to the universities the statutory and regulatory autonomy as provided for in the art. 33 of the Constitution. In those days there has been a great increase in the number of students too, particularly in the University of Milan. While in the academic year 1983-1984, at the beginning of Paolo Mantegazza's rectorship, the registered students were 63.450, in the following years the student number has increased to almost 100.000, thus entailing an intensive work to make suitable the teaching performance and the overall activity for such a huge number of students. Paolo Mantegazza has directed the University trying to carry out this ever-growing task, acting on different fronts. Considering among these the total regulatory framework of the Athenaeum. Actually he has promoted the introduction of a new statute (1996) and the approval of the main rules, implementing the University autonomy. The great growth of the Athenaeum caused different problems: the increasing of the teaching staff to cope with the huge number of students, but new buildings too. In this last regard he did a lot in different areas in the interest of the great part of the Athenaeum faculties. Nevertheless the dimensions reached by the University of Milan required a more far-reaching plan to carry out the 
decongestion of the Athenaeum. He worked towards the doubling of some faculties and the creation of new degrees and new faculties. However it was very soon clear that the most rational solution consisted in the institution of a second Athenaeum in Milan, already assumed when the three-year plan 1991-1993 had been compiled. Thus in 1998 the University of Milan Bicocca was born. In the same year, with the contribution of Paolo Mantegazza and the University of Milan, together with the Pavia University, was instituted the Università dell'Insubria, with branches in Como and Varese. In 1996 was instituted the Vita e salute San Raffaele University too, with a great contribution of the University of Milan during the rectorship of Paolo Mantegazza, transferring there several teachers of the Medicine and surgery Faculty without forgetting the role of Mantegazza as President of Medicine in supporting a University Center in Brescia and the following establishment in 1982 of an independent university. The commitment of Mantegazza for the development of the scientific research in the University has been wide-ranging. At the end of his rectorship the University of Milan was the first Italian university in different international rankings concerning the scientific production. Thanks to these results, at the end of his rectorship in 2002, the University of Milan has been the only University invited to be one of the 12 european universities called up to found the "League of European Research Universities" (best known as LERU) that connects the most prestigious European Universities. During his rectorship he performed his duties (for two mandates) as Vice President of the Rectors Conference of the Italian Universities. He has been a rector of human qualities, with a great operative capability together with a gentle nature. He was sensitive to the student needs, considering the teaching and the student education the first goal of the University. Soon after the end of his Rectorship the then Minister of Education, University and Research, Letizia Moratti, accepted the modification of the Statute of University that in the meantime had been proposed, with the addition of a new article (Art. 56) stating: "In consideration of the merits and the decisive commitment given to the Athenaeum during his rectorship, we confer to Paolo Mantegazza the honouring of Rector Emeritus".

Paolo Mantegazza è stato Rettore dell'Università degli Studi di Milano per quattro mandati consecutivi, dall'anno 1984 al 2001: la più lunga permanenza come Rettore nella storia di questa Università.

E' succeduto a Giuseppe Schiavinato, anch'egli Rettore per molti anni dell'Ateneo milanese. A Paolo Mantegazza, nel 2001, è succeduto come Rettore Enrico Decleva.

In precedenza Mantegazza era stato per 10 anni Preside della Facoltà di Medicina e Chirurgia (dal 1974 al 1984). Aveva pertanto acquisito un'ampia esperienza anche di tipo gestionale.

Il periodo del suo Rettorato è stato un periodo di grandi cambiamenti, nella società (non credo vi sia bisogno di esemplificazioni a questo riguardo), ma anche nell'università. Ricordo che la legge di riforma universitaria, il DPR 382, che ha disposto un nuovo assetto della docenza universitaria ed istituito i ricercatori, nonché i Dipartimenti, è stata 
varata nel 1980; poco tempo dopo è stato istituito il dottorato di ricerca. Nel 1989 la legge n. 168 concesse alle università l' "autonomia" statutaria e regolamentare prevista dall'art. 33 della Costituzione.

Credo che molti ricordino quanti e quali importanti cambiamenti siano avvenuti nella struttura e nel funzionamento delle università e nella stessa vita universitaria in conseguenza di queste leggi di riforma.

In quegli anni si è avuto inoltre il grande aumento della popolazione studentesca, in particolare dell'Università di Milano. Mentre all'inizio del Rettorato di Paolo Mantegazza, nell'anno accademico 1983. 84 , vi erano 63.450 studenti iscritti, il numero di studenti è nel corso degli anni pervenuto ad un numero vicino a 100.000 .

Tutto ciò ha comportato un intenso lavoro per rendere adeguato l'espletamento della didattica e l'attività complessiva dell'Ateneo per un così grande numero di studenti. Paolo Mantegazza ha guidato l'Università tutta per cercare di assolvere questo compito crescente.

Il suo impegno si è esplicato su più fronti, come è naturale in ambito universitario, ma come forse non sempre avviene.

Tra questi anche in relazione al quadro normativo complessivo dell'Ateneo. Infatti egli ha promosso il varo di un nuovo Statuto dell'Ateneo (1996) e furono altresì approvati i principali regolamenti da esso previsti, dando attuazione all'autonomia dell'Ateneo. Ne conseguì una estesa revisione del modo di operare dell'Ateneo, con rilevanti innovazioni in molti ambiti. Ne menziono uno, allora particolarmente significativo: per la prima volta venivano ammessi a far parte del Senato Accademico e del Consiglio di Amministrazione rappresentanti del personale amministrativo e tecnico ed altresì rappresentanti degli studenti. Gli stessi rappresentanti venivano inoltre ammessi al voto per l'elezione del Rettore.

La crescita dell'Ateneo pose problemi di diversa natura: di aumento delle dimensioni del corpo docente, per far fronte al grande numero di studenti, ma anche di nuove necessità edilizie.

Le sue realizzazioni su quest'ultimo fronte sono state molto numerose. Mi limito a menzionare le più rilevanti, anche se non in ordine cronologico, perché danno un'idea dell'ampliamento che l'Ateneo ha avuto in quel periodo:

- Il restauro (con un finanziamento SNAM) del Cortile centrale della Ca' Granda, in via Festa del Perdono, sede centrale dell'Ateneo, e dei loggiati quattrocenteschi del Filarete;

- L'utilizzazione degli spazi della Crociera e della Sottocrociera in via 
Festa del Perdono come biblioteca e sala di consultazione e la realizzazione di un insediamento nella stessa Crociera;

- L'acquisto degli edifici di via Mercalli - Santa Sofia, ora sede di uffici e di attività delle Facoltà umanistiche;

- L'acquisto e la ristrutturazione del Palazzo Resta-Pallavicino di via Conservatorio per la Facoltà di Scienze politiche (in questa occasione ebbe un ruolo importante Piero Bassetti, allora Presidente di Regione Lombardia);

- Il recupero dell'edificio di P.za S. Alessandro - P.za Missori (in precedenza sede del Liceo Beccaria), per il Polo linguistico della Facoltà di Lettere e Filosofia;

- L'affittanza, tuttora in corso, (dalla Curia di Milano) del Palazzo Greppi di via S. Antonio, contenente la Sala Napoleonica, sede di eventi di rappresentanza, e di molti uffici dell'Ateneo;

- La realizzazione di un edificio in via Mangiagalli - via Colombo per le Facoltà di Agraria e di Farmacia;

- La costruzione di un ulteriore edificio per l'ampliamento del Dipartimento di Fisica, in via Celoria - via Ponzio;

- La costruzione di un grande edificio in via Golgi per la Chimica, la Facoltà di Farmacia e per la didattica di diverse Facoltà di Città Studi (conosciuto come "La balena bianca");

- La realizzazione di un edificio in via Trentacoste per le Facoltà di Farmacia e Medicina veterinaria;

- La realizzazione del palazzo LITA a Segrate, in collaborazione con il CNR;

- L'insediamento di Scienze dell'Informazione in via Comelico e successivamente l'insediamento di Scienze dell'Informazione a Crema;

- La realizzazione degli edifici del primo triennio del corso di laurea in Medicina e Chirurgia presso l'Ospedale Sacco a Vialba, l'Ospedale San Paolo e l'Ospedale di Monza;

- Il polo di Veterinaria a Lodi, con l'ospedale per grandi animali;

- L'ampliamento dell'edificio di via Pascal per l'Istituto di Microbiologia e Virologia;

- In seguito ad un accordo con il Comune di Milano, la realizzazione del Nuovo grande Orto Botanico in fregio alla via Golgi, nel terreno retrostante la cosiddetta "Cascina Rosa" a Città Studi.

Come si può notare, si è trattato di realizzazioni in ambiti molto diversi, nell'interesse della maggior parte delle Facoltà dell'Ateneo.

E tuttavia le dimensioni raggiunte dall'Università di Milano 
richiedevano un piano di più ampia portata per attuare il decongestionamento dell'Ateneo, in considerazione dell'altissimo numero di studenti iscritti. Ricordo che allora, per fare un esempio, molte lezioni della Facoltà di Giurisprudenza si tenevano al Teatro Carcano.

Si puntò sul raddoppio di alcune Facoltà e sull'istituzione di nuovi corsi di laurea e di nuove Facoltà. Fu tuttavia ben presto chiaro che la soluzione più razionale consisteva nella prospettiva di un secondo Ateneo statale a Milano, ipotizzato già in occasione della redazione del Piano triennale 1991-1993, dal quale è tratta questa citazione: "sarà compito successivo definire tempi e modi dell'eventuale costituzione d'una vera e propria seconda Università Statale milanese, non come ricalco dell'università esistente, ma prevalentemente come aggregazione di settori disciplinari nuovi, specifici e ben caratterizzati, dotati come tali di autonoma capacità di richiamo, offrendo idonee garanzie di sviluppo anche ai rispettivi settori di ricerca". Per tale secondo Ateneo a Milano venne individuata come sede l'area della Pirelli-Bicocca.

A questo fine occorrevano chiaramente finanziamenti straordinari. Mantegazza racconta che si recò a Roma dal ministro Ruberti e questi gli disse che finanziamenti straordinari sarebbero arrivati se si fosse sdoppiato l'Ateneo.

Fu uno dei casi in cui Mantegazza mise a frutto la sua grande capacità di costruire rapporti di collaborazione con i Ministri che si sono succeduti nel corso del tempo (in particolare Antonio Ruberti, Luigi Berlinguer), nonché con i Direttori del Ministero. Ottimi rapporti di collaborazione ebbe anche con Regione Lombardia.

Nacque così, nel 1998, l'Università di Milano Bicocca. Mantegazza salutò questa nascita con l'augurio che fosse "non soltanto una nuova università, ma una università nuova”.

Se questo fu un evento di grande rilievo, non fu certo il solo. Infatti, nello stesso anno venne istituita anche l'Università dell'Insubria, con sedi a Como e Varese. A tale istituzione Paolo Mantegazza e l'Università di Milano contribuirono, insieme con l'Università di Pavia.

Nel 1996 venne istituita anche l'Università Vita e Salute San Raffaele, alla cui crescita l'Università di Milano aveva notevolmente contribuito durante il rettorato di Paolo Mantegazza, distaccando in quella sede numerosi docenti della Facoltà di Medicina e Chirurgia.

Entrambi gli Atenei di Milano Bicocca e Insubria vennero istituiti durante l'ultimo mandato rettorale di Paolo Mantegazza. Questa fu una delle principali ragioni per le quali Mantegazza ritenne di dover mante- 
nere il suo ruolo di Rettore per così lungo tempo: per portare a termine progetti di così grande rilievo e così a lungo perseguiti.

Senza dimenticare il ruolo che Mantegazza aveva avuto come Preside di Medicina nel favorire un insediamento universitario a Brescia e la successiva costituzione, nel 1982, di una università autonoma.

Dagli anni '80 ai primi anni del 2000 si è attuato un rilevantissimo arricchimento del panorama universitario lombardo e Paolo Mantegazza ha contribuito come nessun altro a tale risultato.

$\mathrm{Ma}$ è opportuno aggiungere qualche altra considerazione. Medico, farmacologo, Preside a lungo di Medicina, Mantegazza si distinse per la disponibilità a tenere conto delle esigenze di tutte le aree scientifiche presenti nell'ateneo, avvertendo in particolare come le biblioteche e le loro dotazioni fossero, per gli umanisti, l'equivalente dei laboratori e delle grandi apparecchiature per i cultori delle scienze sperimentali. Si spiegano in questa prospettiva le grandi sale di consultazione realizzate nella Crociera e nel Sottocrociera di via Festa del Perdono; e si spiegano in questa prospettiva alcune acquisizioni di grande rilevanza come quelle del Papiro di Posidippo e della Biblioteca di Egittologia appartenuta ad Elmar Edel, costituita da 9.000 volumi di particolare pregio.

L'impegno di Mantegazza per lo sviluppo della ricerca scientifica in ateneo e per la creazione di condizioni più idonee, a tutti i livelli, per l'assolvimento dei compiti relativi è stato davvero a tutto campo. Non per niente, nel nuovo Statuto, prima menzionato, venne istituita la Commissione di Ateneo per la ricerca scientifica, composta con rappresentanti di tutte le Facoltà, con il compito di promuovere la ricerca. La relativa disponibilità di risorse consentì d'altro canto in quegli anni di dare un notevole impulso allo svolgimento delle attività relative, potendo contare su fondi europei, del Ministero, della benemerita Fondazione Cariplo e di altri enti ed altresì in misura rilevante anche da parte di aziende. Anche il cosiddetto "conto terzi" vide in quegli anni un significativo incremento.

Alla scarsità dei finanziamenti pubblici (che Mantegazza riuscì in ogni caso a far aumentare) si dovette fare fronte incrementando la contribuzione studentesca. E, bisogna dire, con proteste molto limitate, anche perché si stabilì che una quota delle risorse così ottenute dovesse andare obbligatoriamente a interventi finalizzati a migliorare la qualità della didattica e dei servizi per gli studenti. Le nuove sale di studio create nella sede centrale furono ad esempio finanziate in questo modo. 
Al termine del Rettorato di Paolo Mantegazza l'Università di Milano risultava essere la prima università italiana in diverse classifiche internazionali relative alla produzione scientifica, in particolare in quella del Centro per Studi di Scienza e Tecnologia (CWTS) della Leiden University.

Fu principalmente sulla base di tali risultati che, al termine del Rettorato di Paolo Mantegazza, nell'anno 2002, l'Università di Milano, unica università italiana, veniva invitata ad essere una delle dodici università europee chiamate a costituire la "League of European Research Universities" (più nota con il suo acronimo: LERU), che collega tra loro Università europee di grande prestigio e che svolge ancora oggi un ruolo di primo piano in ambito europeo nella politica universitaria relativa alla ricerca.

Non va altresì dimenticato che durante il suo Rettorato egli ricoprì (per due successivi mandati) anche il ruolo di VicePresidente della Conferenza dei Rettori delle Università Italiane.

Vorrei anche menzionare che una importante caratteristica del suo Rettorato è stata quella di riuscire ad operare suscitando la preziosa collaborazione degli Organi accademici e in una notevole unità di intenti con molti Colleghi chiamati a far parte di Commissioni rettorali.

Nell'ultimo anno del suo Rettorato, in occasione delle votazioni che elessero il suo successore, il Corriere della Sera pubblicò una lunga intervista di Gian Guido Vecchi a Paolo Mantegazza. Fu l'occasione per ripercorrere alcuni momenti salienti del suo operare, ma anche per rivelare aspetti del suo carattere, come da una risposta che diede all'intervistatore, che gli chiedeva se si sarebbe fatto costruire un monumento, analogamente ad alcuni suoi predecessori. Ecco la sua risposta: "Mah, non sono stato abituato alla cultura dell'immagine: dalle mie parti, a Rasa, sopra Varese, ha ancora un profondo significato il motto degli alpini: tas e tira, lavora e taci.....".

Desidero ora ricordare un episodio che mi è caro, relativo al mio rapporto con Paolo Mantegazza, e che rivela la sua attenzione per le proposte che gli giungevano dai docenti. Quando vennero istituiti i Dipartimenti, secondo quanto previsto dal DPR 382, l'Istituto che allora dirigevo, l'Istituto di Genetica, venne accorpato in un grande Dipartimento, che comprendeva la maggior parte delle discipline biologiche della Facoltà di Scienze matematiche, fisiche e naturali. Mi apparve ben presto chiaro che tale accorpamento costituiva un limite alle possibilità di crescita di un gruppo di docenti e ricercatori molto 
attivi, operanti in un ambito ricco di prospettive. Diversamente dal Rettore Schiavinato, al quale $\mathrm{mi}$ ero rivolto in precedenza, Paolo Mantegazza, al quale mi rivolsi nel 1984, subito dopo la sua elezione a Rettore, accolse immediatamente le ragioni della mia richiesta di costituire un Dipartimento autonomo. Nacque così, nel 1985, il Dipartimento di Genetica e di Biologia dei microrganismi, che divenne uno dei Dipartimenti maggiormente attivi dell'Ateneo.

Paolo Mantegazza fu un Rettore di notevoli qualità umane, con una grande capacità operativa, unita ad un carattere mite. Soleva dire che essere Rettore rappresentava un'opportunità unica dal punto di vista umano ed un arricchimento personale, legato anche al fatto che $\mathrm{i}$ Colleghi lo facevano partecipe dei loro problemi, dal momento che suscitava la loro fiducia.

Ebbe una grande attenzione agli studenti ed alle loro esigenze, considerando che l'insegnamento e la formazione degli studenti fossero lo scopo primario dell'Università. Non è mai stato lontano da loro, ed anzi cercava occasioni di incontro e di discussione con loro.

Nel 1994, settantesimo anniversario della fondazione dell'Università degli Studi di Milano, che era anche il decimo anniversario della sua assunzione alla carica di Rettore, Paolo Mantegazza promosse la pubblicazione di un volume che ripercorresse la storia dell'Ateneo.

Nella prefazione di tale volume, si legge: ......"le pagine che seguono vedono la luce in una fase quanto mai delicata della vita italiana. Cresce sempre più la competizione internazionale e le sorti stesse del Paese appaiono legate in non piccola misura alla sua capacità di generare e governare l'innovazione, promuovendo la formazione delle risorse umane e lo sviluppo della ricerca e delle tecnologie in alcuni almeno dei settori strategicamente decisivi, senza con questo perdere di vista le radici storiche e i valori di fondo, non formalisticamente assunti, della tradizione culturale cui apparteniamo."

Credo che queste affermazioni (scritte oltre 20 anni or sono) rivelino il lucido pensiero di Paolo Mantegazza e la sua capacità di interpretare la realtà ed insieme anticipare il futuro.

Forse alcuni ricordano come, poco dopo la conclusione del suo Rettorato, si sia tenuta in aula magna una cerimonia in suo onore. In quell'occasione l'allora Ministro dell'Istruzione, dell'Università e della Ricerca, Letizia Moratti, inviò un messaggio di saluto, ringraziamento e congratulazioni, comunicando di avere accolto la modifica dello Statuto dell'Università che nel frattempo era stata proposta, con l'ag- 
giunta di un nuovo articolo (Art. 56), che così recita: "In considerazione dei meriti acquisiti e dell'impegno determinante profuso per l'Ateneo nel corso del suo rettorato, al prof. Paolo Mantegazza è conferito il titolo onorifico di Rettore emerito". 